\title{
FIB-Milled Nanopore Sensors for Tracking Virus Assembly
}

\author{
Zachary D. Harms ${ }^{1}$, Lye Siang Lee ${ }^{2}$, Adam Zlotnick ${ }^{2}$ and Stephen C. Jacobson ${ }^{1}$ \\ 1. Department of Chemistry, Indiana University, Bloomington, IN USA \\ 2. Department of Molecular and Cellular Biochemistry, Indiana University, Bloomington, IN USA
}

Virus assembly is a coordinated process in which hundreds of subunits react to form complex, symmetric particles [1]. Single-particle methods that examine the mechanisms by which capsid reactions occur are lacking, and measurement of low-abundance intermediates on path to forming complete particles is particularly challenging. We are using resistive-pulse sensing as a label-free, nondestructive technique to characterize the assembly of Hepatitis B Virus Cp149 dimers into $\mathrm{T}=3$ and $\mathrm{T}=4$ icosahedral capsids. This single-particle method counts and sizes intermediates and capsids in real time, with single-particle sensitivity, and at biologically relevant concentrations [2].

A dual-beam focused ion beam (FIB)-scanning electron microscope (SEM) is used to fabricate and characterize the nanopore sensors. Two V-shaped microchannels are first patterned by UV photolithography and etched into a glass substrate. The FIB is then used to mill three nanochannels into the glass substrate to span the two microchannels as shown in Figure 1. A smaller beam current is used to mill conduits that connect each nanochannel segment to create the pores. Each nanochannel contains two pores in series such that each particle is detected twice. To reduce charging during FIB patterning, the substrate is either coated with $120 \mathrm{~nm}$ of $\mathrm{Cr}$ or the glass is milled directly with the aid of an electron flood gun. After milling, the substrates are bonded to glass cover slips to seal the fluidic channels.

For the resistive-pulse sensing measurements, the device is filled with $50 \mathrm{mM}$ HEPES buffer containing $1 \mathrm{M} \mathrm{NaCl}$. Hepatitis B Virus dimers or capsids are placed in one of the buffer-filled reservoirs and are driven by a pressure or potential difference through the bridging nanochannel. Each current pulse corresponds to the transit of one particle through one nanopore, and the pulse amplitude is proportional to particle volume. A histogram of the current displacements $(\Delta \mathrm{i} / \mathrm{i})$ from an assembly reaction at $0.9 \mu \mathrm{M}$ dimer is shown in Figure 2. The smaller $\mathrm{T}=3$ capsids displaced $0.34 \%$ of the baseline current, whereas the larger $\mathrm{T}=4$ capsids displace $0.49 \%$.

Assembly reactions reveal three distinct patterns below, near, and above the pseudo-critical dimer concentration, at which the dimer and capsid concentrations are approximately equal. At dimer concentrations as low as $50 \mathrm{nM}$, well below the pseudo-critical dimer concentration of $0.5 \mu \mathrm{M}$, the early steps of assembly are rate- and product-limiting, and we observe a switch in the ratio of $\mathrm{T}=3$ to $\mathrm{T}=4$ capsids. In contrast, far above the pseudo-critical dimer concentration, incomplete particles assemble rapidly, become kinetically trapped, and slowly anneal into $\mathrm{T}=4$ capsids. These single-particle measurements have provided new insights into assembly paths and energetics.

\section{References:}

[1] A Zlotnick and S Mukhopadhyay, Trends Microbiol. 19 (2011), p. 14-23.

[2] ZD Harms, L Selzer, A Zlotnick and SC Jacobson, ACS Nano 9 (2015), p. 9087-9096. 


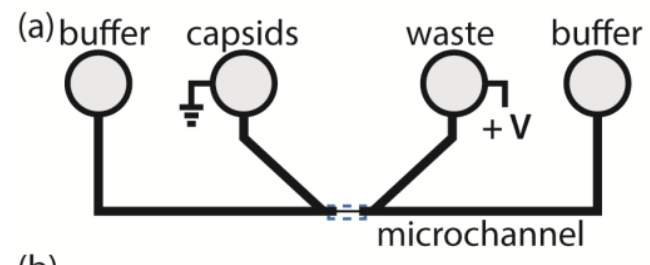

(b)
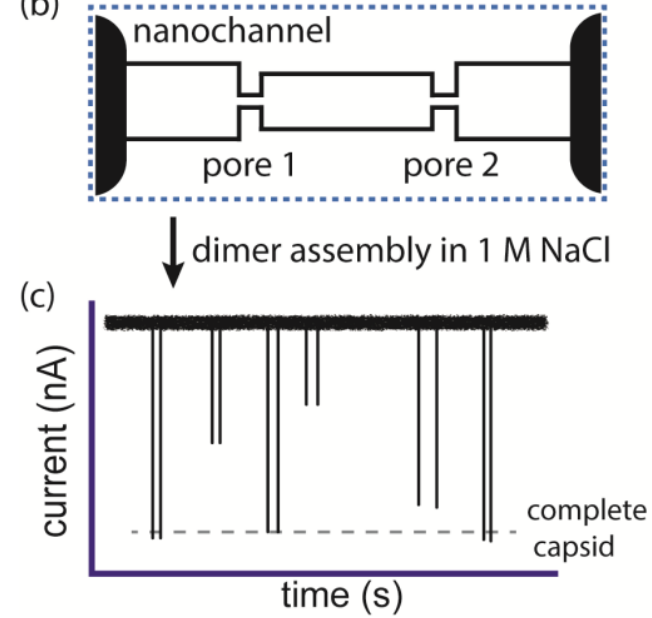

Figure 1. Schematics of the device used for sensing and illustration of sensing data. (a) Two V-shaped microchannels are separated by a $10 \mu \mathrm{m}$ gap. (b) A nanochannel with two pores in series is patterned by FIB milling to connect the two microchannels. (c) Current through the nanochannel is recorded as a function of time. Each particle transiting through the nanochannel produces two decreases in current, or pulses, that are proportional to particle size.

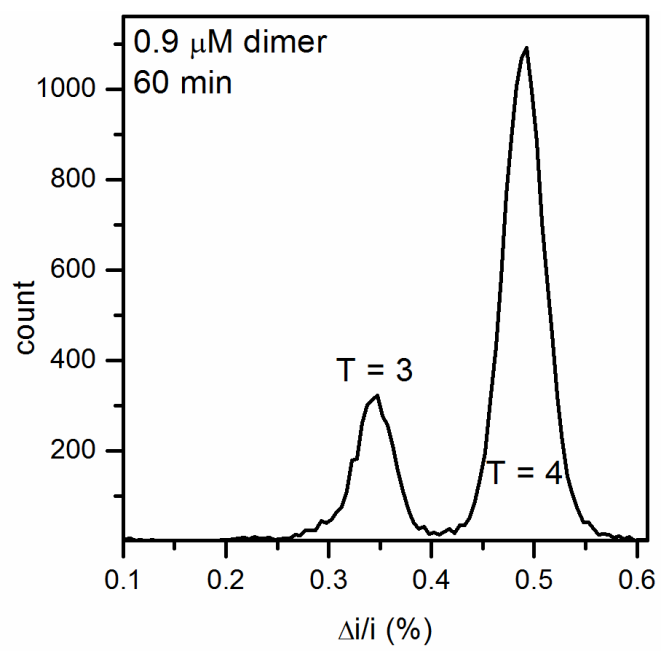

Figure 2. Histogram of Hepatitis B Virus assembly products. A Cp149 dimer concentration of $0.9 \mu \mathrm{M}$ is assembled in $50 \mathrm{mM}$ HEPES buffer with $1 \mathrm{M} \mathrm{NaCl}$. The $\mathrm{T}=3$ capsids displaced $0.34 \%$ of the baseline current and the $\mathrm{T}=4$ capsids displace $0.49 \%$ of baseline current. 